\title{
Quantitative analysis of the applicability limits of the model of a one-dimensional infinite square well
}

\author{
Vladimir Ivchenko*1] \\ ${ }^{1}$ Kherson State Maritime Academy, Department of Natural Sciences Training, Kherson, Ukraine
}

Received on August 22, 2019. Revised on September 16, 2019. Accepted on September 17, 2019.

\begin{abstract}
We discuss the influence of several factors on the deviations from energy spectrum of an infinite square quantum well (QW) for real microscopic systems that can be approximately modelled using particle in a box. We introduce the "blurring" potential in the form of the modified Woods-Saxon potential and solve the corresponding Schrödinger equation. It is found that the increase of the degree of blurring $\delta$ of the QW leads to the increase of number of the energy levels inside it and to increase of deviations from the quadratic dependence $\varepsilon(n)$ ( $\varepsilon$ is the particle energy, $n$ is the energy level number) typical for the infinite square QW, especially, for the energy levels close to the QW "tops". It is most surprising that for relatively "large" values of $\delta$ the difference between the levels energies of such well and the appropriate (with the same $n$ ) levels energies of the square QW with the same depth changes sign (from positive to negative) as number $n$ increases. We also conclude that the asymmetry of the QW and non-equality $m_{\mathrm{in}} \neq m_{\mathrm{out}}$ (where $m_{\mathrm{in}}$ and $m_{\mathrm{out}}$ are the particle effective mass inside and outside the QW) play a significant role for the relatively "shallow" well near the QW top.
\end{abstract}

Keywords: infinite square quantum well, the "blurring" potential, the asymmetry of QW, the position-dependent effective mass.

\section{Introduction}

As it is known, the study of model systems, for which there are simple analytic solutions of the time independent Schrödinger equation, makes it possible to understand the methods of quantum mechanics more comprehensively. In addition, the results obtained are of independent interest, since they reflect, in some approximation, the properties of the corresponding real systems. One of such idealized system is the particle in a onedimensional box model [1], 2] (also known as the infinite square quantum well $(\overline{\mathrm{QW}})$ ) that describes a particle which can only move freely along a linear segment of finite length. Inside this segment the potential is considered equal to zero. At all other points of the straight line the potential goes to infinity. The particle in a box model is mainly used as an approximation for the description of quite complicated quantum systems. For example, the behaviour of electrons in some chemical compounds can be precisely modelled using particle in a box 3 .

The energy spectrum of such QW is very simple [1]:

$$
E=\frac{\pi^{2} \hbar^{2}}{2 m L^{2}} n^{2}
$$

where $\hbar$ is the reduced Planck constant, $m$ is the mass of the particle, $L$ is the QW width, $n$ is a positive integer $(1,2,3,4 \ldots)$. The wave functions of the problem vanish everywhere beyond and at the QW edges. These solutions of the Schrödinger equation are alternatively even and

*Correspondence email address: reterty@gmail.com odd with respect to the center of the potential well (this will be true for any symmetric potential). The even parity solutions (cosines) correspond to odd $n$ numbers whereas the odd parity solutions (sinuses) correspond to even $n$ numbers 1].

There are many articles on square quantum wells (finite or infinite) in the literature. The educational papers in this area can be divided into the groups of works devoted to: improving the procedures for approximate finding energy spectrum in the finite square QW 4 8]; solving the time-dependent Schrödinger equation in the case of a one-dimensional square QW with moving walls 9,10 ; the study of symmetry properties of the quantum systems with square-well potential [11,12]; the investigations of the relativistic particle in a box 13,14$]$; the considerations of boundary conditions for an infinite square-well potential in quantum mechanics 15, 16 et al. However, from the theoretical point of view two issues are to be clarified. What are the possible reasons for the deviations from the model of a one-dimensional infinite square QW in reality? How do these factors transform the energy spectrum (1)?

There are several factors that cause the deviations from the infinite square QW model for real microscopic systems. These are:

1. The finite QW depth.

2. The small "blurring" of the QW walls.

3. The asymmetry of QW.

4. The position-dependent effective mass in solids. 
5. Existence of two additional mechanical degrees of freedom of the particle.

The aim of this article is the consideration of the influence of such factors on the deviations from energy spectrum (1) in the real QW systems (heterostructures, quantum dots and atomic nuclei, etc.) that can be approximately modelled using particle in a box.

\section{The "blurring" of the walls and finite quantum well depth}

The assumption of the vertical walls is non-physical because the force $F_{x}=-\partial V / \partial x$ turns to infinity in their locations. Various reasons can lead to the formation of a finite slope of the potential profile in the QW, namely: mutual diffusion of the components through the phase interface (a boundary between two solids) in heterostructures with the formation of a "thin transition layer"; unevenness ("roughness") of the interface itself; formation of a double electric layer near the interface. The mismatch (difference) of the lattice constants may result in mechanical stresses that will decay as one moves away from the heterointerface distorting the rectangular shape of the well. The finite QW depth is caused by the finite difference between the work functions of a particle inside and outside a well.

Let us consider the finite "blurring" potential in the form of the modified Woods-Saxon potential [17,18 that represents a square well with rounded edges:

$$
\begin{aligned}
& V(y)=\frac{V_{0}(1+\exp (1 / \delta))}{\exp (1 / \delta)} \\
& \times\left[\frac{\exp \left(\frac{y-1}{\delta}\right)}{1+\exp \left(\frac{y-1}{\delta}\right)}-\frac{1}{1+\exp (1 / \delta)}\right],
\end{aligned}
$$

where $V_{0}$ is the QW depth; $y=|x| /(L / 2)$ is the dimensionless coordinate, expressed in terms of half-width of the QW $L / 2 ; \delta=\Delta /(L / 2) \ll 1$. Here $\Delta$ is the quantity characterizing the degree of blurring of the QW walls (the thickness or diffuseness of the QW wall). The depth of the QW (2) is strictly fixed and precisely equal to $V_{0}$, whereas the depth of the Woods-Saxon QW in its original form 17] depends on the value of $\delta$. Such a symmetric potential $V(y)$ has two inflection points, which always correspond to the boundaries of the quantum system $(x= \pm L / 2)$. For $y \ll 1$ we have: $V(y) \approx V_{0} y / \delta[1+\exp (1 / \delta)]$. In the case $y \rightarrow \infty V(y) \approx V_{0}[1-(1+\exp (1 / \delta)) \exp (-y / \delta)]$, that is, asymptotically tends to $V_{0}$ (see solid line in Fig. 1 ). If $V_{0} \rightarrow \infty, \delta \rightarrow 0$, the potential (2) is transformed into the potential of the infinite square QW (dashed line in Fig. 1). The QW in form (2) is considered for the first time.

We write the Schrödinger equation for (2) as

$$
\frac{\mathrm{d}^{2} \psi(y)}{\mathrm{d} y^{2}}+\left[k^{2}-q^{2} \frac{\exp \left(\frac{y-1}{\delta}\right)}{1+\exp \left(\frac{y-1}{\delta}\right)}\right] \psi(y)=0,
$$

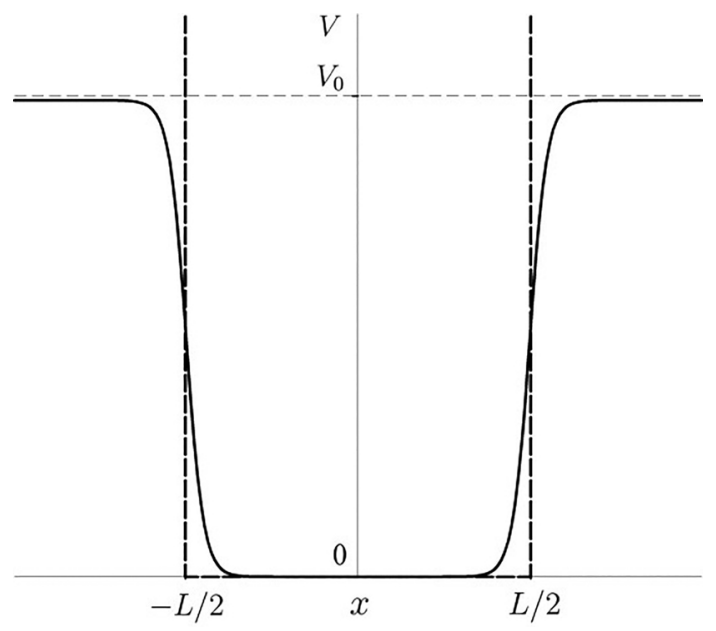

Figure 1: The solid line is the "blurring" quantum well (2). The dashed line is the infinite square quantum well.

where

$$
\begin{gathered}
k=\frac{\pi}{2} \sqrt{\frac{\varepsilon+\exp (-1 / \delta)}{u},} \\
q=\frac{\pi}{2} \sqrt{\frac{1+\exp (1 / \delta)}{u \exp (1 / \delta)}}>k,
\end{gathered}
$$

$\varepsilon=E / V_{0}(0<\varepsilon<1)$ is the dimensionless energy of the particle; $u=\pi^{2} \hbar^{2} /\left(2 m L^{2} V_{0}\right)$ is the dimensionless QW depth. We search the solutions of Eq. (3) that satisfy the Dirichlet condition: $\lim _{y \rightarrow \infty} \psi(y)=0$. Moreover, since the potential (2) is symmetric, Eq. (3) will have definite parity even/odd solutions. It is worth mentioning that the discontinuity at $x=0$ in the slope of $V(y(x))$ is finite, so $\psi(y(x))$ is continuous, and so is its slope. It is therefore unnecessary to consider the boundary at $x=0$, and to have two regions $x \leq 0$ and $x>0$.

To satisfy the Dirichlet condition, we represent the wave function in the form: $\psi(y)=\varphi(y) \exp \left(-\sqrt{q^{2}-k^{2}} y\right)$. If we introduce additionally new variable

$$
z=-\exp \left(\frac{1-y}{\delta}\right)
$$

then the Schrödinger Eq. (3) can be transformed to the Euler's hypergeometric differential equation [19]:

$z(1-z) \frac{\mathrm{d}^{2} \varphi}{\mathrm{d} z^{2}}+\left(1+2 \delta \sqrt{q^{2}-k^{2}}\right)(1-z) \frac{\mathrm{d} \varphi}{\mathrm{d} z}-\delta^{2} q^{2} \varphi=0$.

The solution of this equation is the Gauss's hypergeometric function ${ }_{2} F_{1}(a, b ; c ; z)[19]$ with

$a=\delta\left(\sqrt{q^{2}-k^{2}}+i k\right), b=\bar{a}, c=1+2 \delta \sqrt{q^{2}-k^{2}}$.

Therefore, the even parity solution of our problem is

$$
\begin{aligned}
& \psi_{\mathrm{e}}(y)=C_{2} F_{1 \varepsilon_{n}}(a, b ; c ; z) \exp \left(-\sqrt{q^{2}-k^{2}} y\right), \\
& n=0,2,4, \ldots
\end{aligned}
$$


where $C$ is the normalizing factor and

$$
\left.\frac{\mathrm{d} \psi_{\mathrm{e}}}{\mathrm{d} y}\right|_{y=0}=0 .
$$

The odd parity solution is given as

$$
\begin{aligned}
& \psi_{\mathrm{o}}(y)=C \operatorname{sgn}(x){ }_{2} F_{1 \varepsilon_{n}}(a, b ; c ; z) \exp \left(-\sqrt{q^{2}-k^{2}} y\right), \\
& n=1,3,5, \ldots
\end{aligned}
$$

where

$$
\psi_{\mathrm{o}}(0)=0
$$

The conditions (9), (11) quantize energies $\varepsilon_{n}$, where quantum number $n$ numbers the energy levels in order of increasing their energy.

Fig. 2, 3 show the results of numerical calculations (for the numerical solution of the transcendental equations hereinafter we apply the "fsolve" command of the Maple software [20]) of particle energy $\varepsilon$ depending on continuous variable $n$, based on Eqs. (4-11), at different values of $\delta$ and $u$. It should be noted that in Eqs. (8) and (10) $n$ is the integer variable. But we let it change continuously to get smooth curves $\varepsilon(n)$.

From Fig. 2 it is seen that taking into account the nonzero value of $\delta$ will cause the deviations from quadratic dependence $\varepsilon(n)$ typical for the infinite square QW (see Eq. (1)), especially, for the energy levels close to the QW "tops". With an increase in the degree of blurring of QW $\delta$, the number of the energy levels inside it increases too. It is most surprising is that for relatively "large" values of $\delta$ the difference between the levels energies of such well and the appropriate (with the same $n$ ) levels energies of square QW with the same depth changes sign (from positive to negative) as number $n$ increases. The above results are not previously reported in literature and can help readers to probe the limits of applicability of the square (infinite or finite) QW.

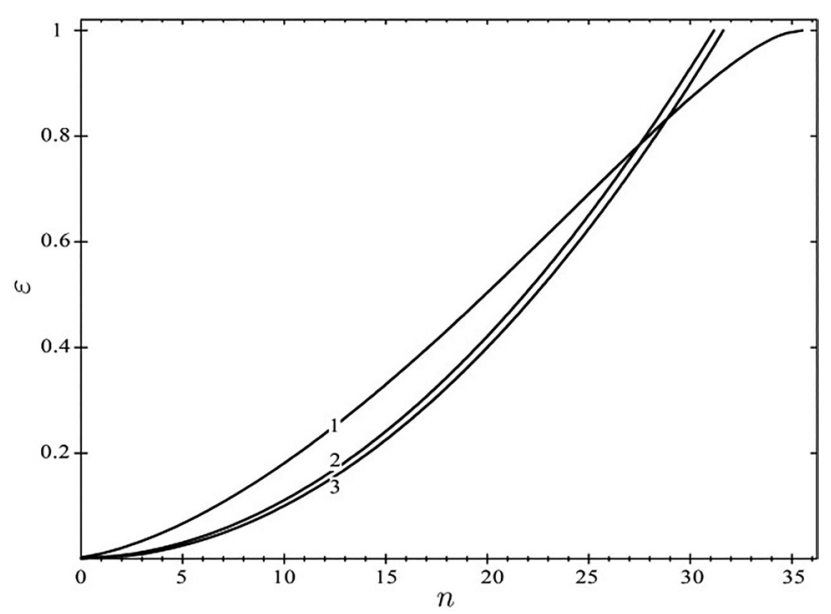

Figure 2: Dimensionless energy $\varepsilon$ as a function of continuous variable $n$. (1) $u=10^{-3}, \delta=0.1$; (2) $u=10^{-3}, \delta=10^{-3}$; (3) $\varepsilon=u n^{2}$, where $u=10^{-3}$.
As it expected the decrease of a well depth (that is the increase of the dimensionless quantity $u$; see Fig. 3) causes the decrease of number of such discrete states. Therefore, there always exist at least one discrete level located near the top of QW (2) as for the case of the symmetric finite square QW. This level corresponds to the even parity solution (8) with $n=0$.

Our numerical calculations (which are done using Eq. (1) and Eq. (12) and putting $\alpha=1$ ) also indicate that the location of the levels within finite and infinite square QW approximations is practically the same (the maximum relative error in the determination of the levels position is less than $5 \%$ ) if $u \approx 10^{-4}$, that is, if square QW depth $V_{0}$ is four orders of magnitude greater than the energy of the ground state (equal to $\pi^{2} \hbar^{2} /\left(2 m L^{2}\right)$ ) of the infinite square QW. This new fact is also important for students' understanding the applicability limits of the model of an infinite square QW.

\section{The asymmetry of quantum well}

The QW, where the inversion symmetry with respect to the quantum well center is broken by some means, is called the asymmetric QW. The symmetry breaking can be achieved in several different ways, for example, varying the material (alloy) composition in QW or applying an electric field along the growth direction 21]. The energy spectrum of such a QW differs from the infinite square QW not only due to the finite well depth but also due to the asymmetry of the potential.

For the simplest case of the asymmetric square QW the transcendental equation for finding particle energy $\varepsilon_{\mathrm{a}}$ has the following form 22 :

$$
\begin{aligned}
& \pi \sqrt{\varepsilon_{\mathrm{a}} / u}+\arcsin \sqrt{\varepsilon_{\mathrm{a}}}+\arcsin \sqrt{\varepsilon_{\mathrm{a}} / \alpha}=\pi n, \\
& n=1,2,3, \ldots
\end{aligned}
$$

where $\varepsilon_{\mathrm{a}}=E / V_{1}\left(0<\varepsilon_{\mathrm{a}}<1\right), u=\pi^{2} \hbar^{2} / 2 m L^{2} V_{1}$; $\alpha=V_{2} / V_{1}>1$ is the factor that defines the degree

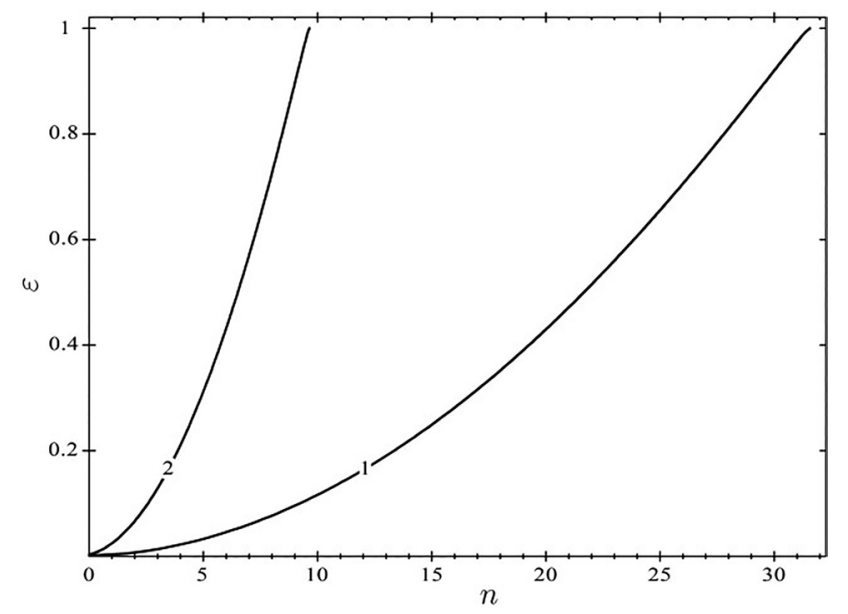

Figure 3: Dimensionless energy $\varepsilon$ as a function of continuous variable $n$. (1) $\delta=10^{-2}, u=10^{-3}$; (2) $\delta=10^{-2}, u=10^{-2}$. 
of well asymmetry; $V_{1}$ and $V_{2}$ are the heights of the left and right QW walls respectively. In Fig. 4 we plot dependences $\varepsilon_{\mathrm{a}}(n)$, constructed at different values of $\alpha$ and $u$ using Eq. (12).

We see that $\varepsilon_{\mathrm{a}}>\varepsilon_{\mathrm{s}}$, where $\varepsilon_{\mathrm{s}}=\varepsilon_{\mathrm{a}}(\alpha=1)$ is the particle energy in the relevant symmetric square $\mathrm{QW}$ $\left(V_{1,2}=V_{0}\right)$. However, the asymmetry of a $\mathrm{QW}$ can play a significant role only for the relatively "shallow" well near the QW top. These obtained features of the energy spectrum are of pedagogical value, since they can grasp the applicability limits of the model of an infinitely deep $\mathrm{QW}$, which is, naturally, always symmetric. We also note that there are always so "large" values of parameters $\alpha$ and $u$ at which even single discrete level does not exist.

\section{The position-dependent effective mass}

In solid state physics, a particle effective mass is the quasimass of a particle that takes into account its interaction with the internal electric field of a condensed matter (crystal). The effective mass of particles in low-dimensional semiconductors is position-dependent. Within the simplest approximation the effective mass inside the well, $m_{\mathrm{in} n}$, is constant but differs from the mass outside the well, $m_{\text {out }}$. To take into account different effective mass inside and outside the well one uses the BenDaniel-Duke boundary conditions 23 describing the continuity of the wave functions and the continuity of the probability currents density at the interfaces. If one chooses the origin at the left QW wall then these conditions will be as follows:

$$
\left\{\begin{array}{l}
\psi_{\mathrm{in}}\left(x_{\mathrm{b}}\right)=\psi_{\text {out }}\left(x_{\mathrm{b}}\right) \\
\left.\frac{\mathrm{d} \psi_{\text {in }}}{\mathrm{d} x}\right|_{x=x_{\mathrm{b}}}=\left.\beta \frac{\mathrm{d} \psi_{\text {out }}}{\mathrm{d} x}\right|_{x=x_{\mathrm{b}}}
\end{array}, x_{\mathrm{b}}=0, L\right.
$$

where $\beta=m_{\mathrm{in}} / m_{\mathrm{out}}$ is the mass discontinuity factor.

We seek solution of the stationary Schrödinger equation in the form:

$$
\begin{gathered}
\psi_{\mathrm{i} n}(x)=C_{\mathrm{i} n} \sin \left(k_{\mathrm{i} n} x+\varphi\right), \\
\psi_{\text {out }}(x)=C_{\text {out }} \exp \left(-k_{\text {out }}|x|\right),
\end{gathered}
$$

where

$$
\left\{\begin{array}{l}
k_{\mathrm{in}}=\sqrt{\frac{2 m_{\mathrm{in}} E}{\hbar^{2}}} \\
k_{\text {out }}=\sqrt{\frac{2 m_{\text {out }}\left(V_{0}-E\right)}{\hbar^{2}}} .
\end{array}\right.
$$

Using Eqs. (13)-(15), we derive:

$$
\left\{\begin{array}{l}
\cot \varphi=\beta \frac{k_{\text {out }}}{k_{\text {in }}} \\
\cot \left(k_{\text {in }} L+\varphi\right)=-\beta \frac{k_{\text {out }}}{k_{\text {in }}}
\end{array} .\right.
$$

Solving system (17) and using Eq. (16), we finally get:

$$
\begin{aligned}
& \pi \sqrt{\varepsilon / u}+2 \arcsin \sqrt{\varepsilon /(\beta+(1-\beta) \varepsilon)}=\pi n, \\
& n=1,2,3, \ldots
\end{aligned}
$$

Eq. (16) obtained here has that one advantage that it describes the energy spectrum of both even and odd states sumultaneously, whereas usually [23] two equations are applied to find eigenvalues .

In Fig. 5 we plot dependences $\varepsilon(n)$, constructed at different values of $\beta$ and $u$ using Eq. (16).

At fixed value of $n$ the particle energy is an increasing function of parameter $\beta$. We see that the deviation of parameter $\beta$ from 1 can play a significant role only for the relatively "shallow" well near the QW top. These obtained features of the energy spectrum are of pedagogical value, since they can grasp the applicability limits of the model of an infinitely deep QW, for which difference between $m_{\mathrm{in}}$ and $m_{\mathrm{out}}$ in no way affects the energy spectrum (see Eq. (18). We also note that there are always so "large" values of parameters $\beta$ and $u$ at which even single discrete level does not exist .

\section{Existence of two additional mechanical degrees of freedom of the particle}

In reality the motion of a particle occurs not in one but in three directions. These two extra motions can be finite

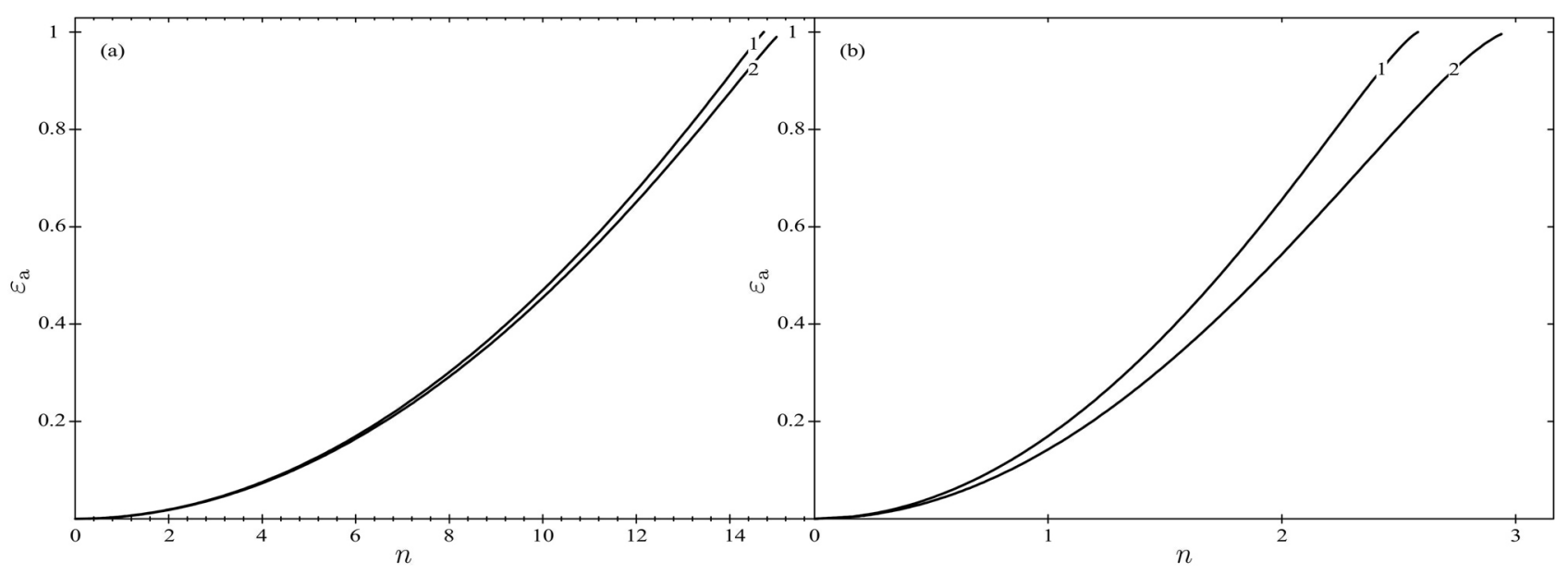

Figure 4: Dimensionless energy $\varepsilon_{\mathrm{a}}$ as a function of continuous variable $n$ for: (1) $\alpha=10$; (2) $\alpha=1$. (a) $u=5 \cdot 10^{-3}$; (b) $u=0.25$. 


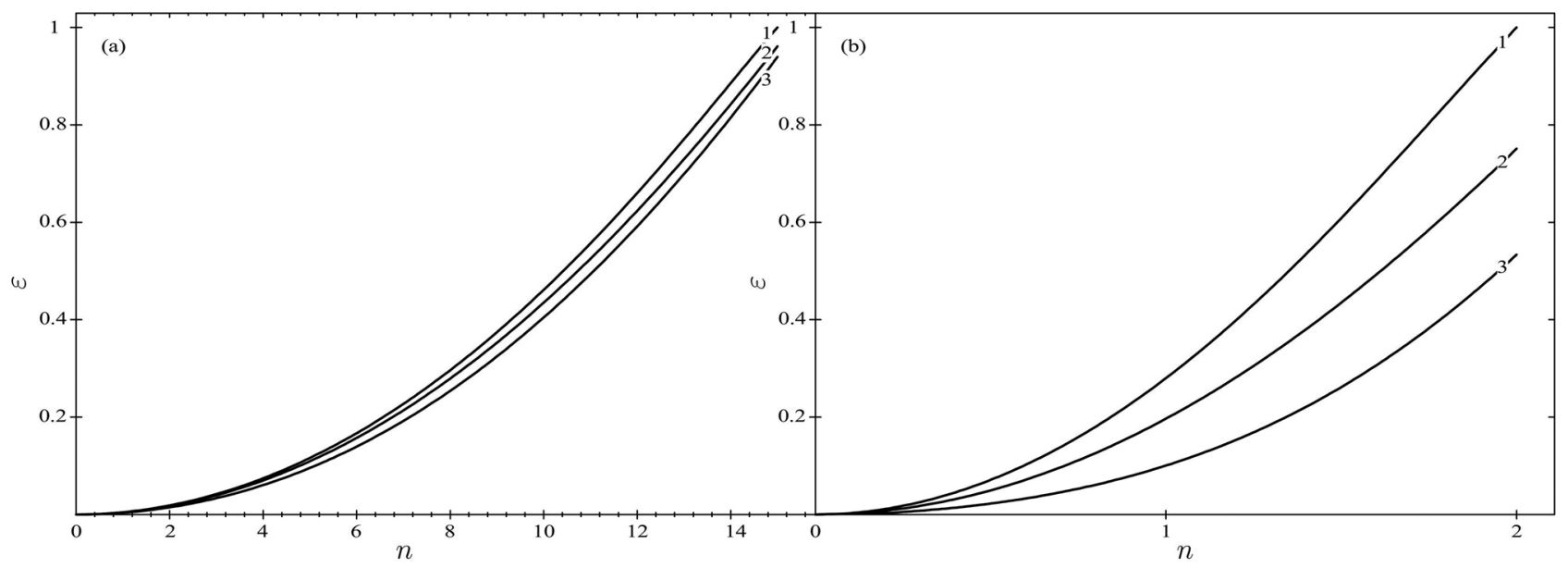

Figure 5: Dimensionless energy $\varepsilon$ as a function of continuous variable $n$ for: (1) $\beta=10$; (2) $\beta=1$; (3) $\beta=0.1$. (a) $u=5 \cdot 10^{-3}$; (b) $u=0.25$.

as in the case of rotational motion in a quantum dot or have a quasi-free character (the so-called lateral motion) as for a two-dimensional nanosystem. As an example let us consider the infinite spherical QW. The position of the energy levels in such a well is determined via the roots of equation 24

$$
J_{l+\frac{1}{2}}\left(\sqrt{\frac{2 m E}{\hbar^{2}}} \frac{L}{2}\right)=0
$$

where $J_{l+\frac{1}{2}}\left(\sqrt{\frac{2 m E}{\hbar^{2}}} \frac{L}{2}\right)$ is the half-integer order Bessel functions of the first kind; $l=0,1,2 \ldots$ is the azimuthal (orbital) quantum number; $L$ is the diameter of $\mathrm{QW}$. Thus, the positions of energy levels in a spherical QW depend on two quantum numbers: orbital $(l)$ and radial $\left(n_{r}\right)$. As a result, the eigenvalue spacing (in contrast to the one-dimensional $\mathrm{QW}$ ) is quite complex in this case [24].

It is interesting that for $l=0$ (that is in the absence of orbital motion of the particle) equation (17) can be solved analytically and we get the energy spectrum in form (1) with only even values of $n_{r}$. Here we explain only why there are no odd values of $n_{r}$ in this spectrum. The point is that in this case the radial wave function can be represented in the form: $\chi_{l}(r) / r$ [24], where $\chi_{l}(r)$ function satisfies the same Schrödinger equation as in the one-dimensional case. Since the radial wave function should take the finite value at $r=0$, then $\chi_{l}(0)=0$. But it is precisely this condition that satisfies the levels with the even $n_{r}$.

Thus, the adding of the new mechanical degrees of freedom to the particle in box problem significantly complicates its solution and the interpretation of results. This circumstance should be explained to students on such and similar examples.

\section{Conclusions}

We discuss the influence of several factors on the deviations from energy spectrum of an infinite square quantum well (QW) for real microscopic systems that can be approximately modelled using particle in a box. We introduce the "blurring" potential in the form of the modified Woods-Saxon potential and solve the corresponding Schrödinger equation. It is found that the increase of the degree of blurring $\delta$ of the QW leads to the increase of number of the energy levels inside it and to increase of deviations from the quadratic dependence $\varepsilon(n)(\varepsilon$ is the particle energy, $n$ is the energy level number) typical for the infinite square QW, especially, for the energy levels close to the QW "tops". It is most surprising that for relatively "large" values of $\delta$ the difference between the levels energies of such well and the appropriate (with the same $n$ ) levels energies of the square QW with the same depth changes sign (from positive to negative) as number $n$ increases. We also conclude that the asymmetry of the QW and non-equality $m_{\mathrm{in}} \neq m_{\mathrm{out}}$ (where $m_{\mathrm{in}}$ and $m_{\mathrm{out}}$ are the particle effective mass inside and outside the QW) play a significant role for the relatively "shallow" well near the QW top.

\section{References}

[1] D.J. Griffths, Introduction to Quantum Mechanics (Prentice Hall, Nova Jersey, 1995), p. 24.

[2] D.F. Styer, Am. J. Phys. 69, 56 (2001).

[3] T. Wimpfheimer, J. Lab. Chem. Educ. 3, 19 (2015).

[4] P.H. Pitkanen, Am. J. Phys. 23, 111 (1955).

[5] B.I. Barker, G.H. Rayborn, J.W. Ioup and G.E. Ioup, Am. J. Phys. 59, 1038 (1991).

[6] B.O.F. Alcantara and D.J. Griffths, Am. J. Phys. 74, 43 (2006).

[7] P.G. Guest, Am. J. Phys. 40, 1175 (1972).

[8] D.W.L. Sprung, H. Wu and J. Martorell, Eur. J. Phys. 13, 21 (1992). 
[9] D.N. Pinder, Am. J. Phys. 58, 54 (1990).

[10] J.G. Cordes, D. Kiang and H. Nakajima, Am. J. Phys. 52, 155 (1984).

[11] F. Leyvraz, A. Frank, R. Lemus and M.V. Andrés, Am. J. Phys. 65, 1087 (1997)

[12] D. Shi-Hai and M. Zhong-Qi, Am. J. Phys. 70, 520 (2002).

[13] C.G. Adler, Am. J. Phys. 39, 305(1971).

[14] P. Alberto, S. Das and E.C. Vagenas, Eur. J. Phys. 39, 025401 (2017).

[15] M. Bowen and J. Coster, Am. J. Phys. 49, 80 (1981).

[16] R. Seki, Am. J. Phys. 39, 929 (1971).

[17] R.D. Woods and D.S. Saxon, Phys. Rev. 95, 577 (1954).

[18] V.K. Dolmatov, J.L. King and J.C. Oglesby, J. Phys. B: At. Mol. Opt. Phys. 45, 105102 (2012).

[19] M. Abramowitz and I.A. Stegun, Handbook of Mathematical Functions with Formulas, Graphs, and Mathematical Tables (Dover, New York, 1972), p. 555.

[20] Maple User Manual, http://www.maplesoft.com/ documentationcenter/maple2017/UserManual.pdf

[21] H.C. Liu and F. Capasso, Intersubband transitions in quantum wells: Physics and device applications I (Academic Press, London, 2000), p. 26.

[22] L.D. Landau and E.M. Lifshitz, Quantum Mechanics: Non-Relativistic Theory (Pergamon Press, 1977), v. 3, $3^{\mathrm{a}}$ ed., p. 63.

[23] V. Barsan and M.C. Ciornei, Eur. J. Phys. 38, 015407 (2017).

[24] S. Flügge, Practical Quantum Mechanics I (SpringerVerlag, New York, 1974). 